TITLE:

\title{
Force density method for simultaneous optimization of geometry and topology of trusses
}

\author{
$\operatorname{AUTHOR}(S)$ :
}

Ohsaki, Makoto; Hayashi, Kazuki

\section{CITATION:}

Ohsaki, Makoto ...[et al]. Force density method for simultaneous optimization of geometry and topology of trusses. Structural and Multidisciplinary Optimization 2017, 56(5): 11571168

ISSUE DATE:

2017-11

URL:

http://hdl.handle.net/2433/229535

\section{RIGHT:}

The final publication is available at Springer via https://doi.org/10.1007/s00158-017-1710-8: The full-text file will be made open to the public on 01 November 2018 in accordance with publisher's 'Terms and Conditions for Self-

Archiving'; This is not the published version. Please cite only the published version.; この論文は出版蝂でありません 。引用の際には出版社版をご確認ご利用ください。 


\title{
Force density method for simultaneous optimization of geometry and topology of trusses
}

\author{
Makoto Ohsaki • Kazuki Hayashi
}

Received: date / Revised version: date

\begin{abstract}
A new method of simultaneous optimization of geometry and topology is presented for plane and spatial trusses. Compliance under single loading condition is minimized for specified structural volume. The difficulties due to existence of melting nodes are successfully avoided by considering force density, which is the ratio of axial force to the member length, as design variable. By using the fact that the optimal truss is statically determinate with the same absolute value of stress in existing members, the compliance and structural volume are expressed as explicit functions of force density only. After obtaining optimal cross-sectional area, nodal locations, and topology, the cross-sectional areas and nodal coordinates are further optimized using a conventional method of nonlinear programming. Accuracy of the optimal solution is verified through examples of plane trusses and a spatial truss. It is shown that various nearly optimal solutions can be found using the proposed method.
\end{abstract}

Keywords Truss - Simultaneous optimization of topology and geometry - Force density method . Compliance

Makoto Ohsaki

Dept. of Architecture and Architectural Engineering, Graduate School of Engineering,

Kyoto University, Japan,

E-mail: ohsaki@archi.kyoto-u.ac.jp

Kazuki Hayashi

Dept. of Architecture and Architectural Engineering, Graduate School of Engineering,

Kyoto University, Japan,

E-mail: hayashi.kazuki.55a@st.kyoto-u.ac.jp

\section{Introduction}

Topology optimization of trusses is a well-established field of research, and various methods including mathematical programming and heuristic approaches have been developed (Ohsaki 2010; Bendsøe and Sigmund 2003). The ground structure approach (Dorn et al. 1964) is generally used for obtaining a sparse truss from a highly connected ground structure. In this process, the nodal locations are fixed; therefore, a dense ground structure is needed to obtain appropriate locations of nodes.

Optimization of nodal locations of trusses is called geometry optimization or configuration optimization. This process is rather easy, if the possible location of each node is restricted in a small neighborhood region of the initial location. When optimizing the geometry of a truss, the member cross-sectional areas are also preferred to be varied, and the thin members after optimization are to be removed. This way, the geometry and topology of a truss can be simultaneously optimized. For a simple truss, optimal geometry and topology may be obtained by solving two optimization problems alternatively by considering cross-sectional areas and nodal coordinates, respectively, as design variables (Dobbs and Felton 1969). However, if the nodes are allowed to move in a wide range of the design space, then so-called melting nodes will exist, where closely spaced nodes are connected by very short members (Ohsaki 1998; Achtziger 2007).

To overcome difficulties for simultaneous optimization of geometry and topology, a growing process has been developed (McKeown 1998; Hagishita and Ohsaki 2009). The process starts with a very simple truss and adds nodes and members sequentially based on some heuristics. Although a sparse optimal topology and geometry can be found, the solution obtained by this 
method does not satisfy any theoretically defined optimality criteria.

The most serious difficulty due to existence of melting nodes is that the axial stiffness of a member becomes infinity, if its length approaches zero. Furthermore, the sensitivity coefficients of stiffness of a very short member with respect to nodal coordinates have very large values, and the sensitivity coefficients are discontinuous when the member length vanishes. The discontinuity can be alleviated by modification of stiffness of short members using sigmoid functions. Guo et al. (2003) proposed a method for finding a globally stable truss by condensing some nodes of a regular truss without using overlapping members.

There are various methods for reformulation of topology optimization problems (Stolpe 2007); however, few studies have been done for problems involving geometry modification. Achtziger (2007) reformulated the problem of simultaneous optimization to a problem with cubic objective function and quadratic constraints. He discussed properties of simultaneous approach, alternating approaches, and implicit programming approach in detail. It is pointed out that the alternating approach does not always lead to the optimal solution of the problem with two types of variable. He proposed a method based on the implicit function approach under condition that each pair of nodes is sufficiently spaced.

In the field of tension structures such as cable nets and tensegrity structures, the force density method is used for finding the self-equilibrium shape (Sheck 1974; Zhang and Ohsaki 2015). The force density is defined as the ratio of axial force to length, and the nodal coordinates at equilibrium are obtained by solving a set of linear equations of the force densities of all members. Descamps and Coelho (2014) used force density as one of design variables in their intermediate problem in the reformulation process. However, they finally solved a problem considering stress, nodal location, and the ratio of cross-sectional area to the member length, which is called section density, as design variables.

In this paper, a new method of simultaneous optimization of geometry and topology is presented for plane and spatial trusses. The difficulties stated above are successfully avoided by considering force density as design variable. The compliance and structural volume are expressed as explicit functions of force density using the fact that the optimal truss is statically determinate with the same absolute value of stress in existing members. Smoothing approximation is used for the absolute value of force density to find optimal solutions using a nonlinear programming (NLP), where the variables are force densities only. The accuracy of the optimal solu-

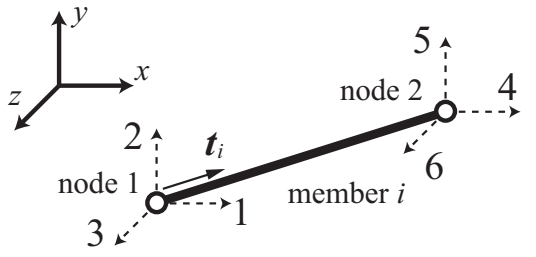

Fig. 1 Definition of member direction vector $\boldsymbol{t}_{i}$.

tion is verified through an example of a plane truss, and an example of spatial truss is also presented.

We consider a truss in two- or three-dimensional space. However, the equations and problems in the following sections are formulated, for generality, for a truss in three-dimensional space. Furthermore, throughout the paper, the $i$ th component of a vector $\boldsymbol{a}$ is written as $a_{i}$ without explanation, and the $(i, j)$ component of a matrix $\boldsymbol{B}$ is denoted by $B_{i j}$. All vectors are assumed to be column vectors.

\section{Equilibrium and stiffness equations}

Consider a truss with $n$ nodes and $m$ members. Let $A_{i}$ and $L_{i}$ denote the cross-sectional area and length of the $i$ th member, respectively. The global coordinates $(x, y, z)$, local node numbers, and local displacement numbers are defined in Fig. 1 . Let $\boldsymbol{t}_{i} \in \mathbb{R}^{3}$ denote the unit vector directed from node 1 to 2 of the $i$ th member; i.e. the three components of $\boldsymbol{t}_{i}$ correspond to the directional cosines with respect to $x$-, $y$-, and $z$-axes, respectively. A vector $\boldsymbol{d}_{i} \in \mathbb{R}^{6}$ is defined as

$\boldsymbol{d}_{i}=\left(-\boldsymbol{t}_{\boldsymbol{i}}^{T}, \boldsymbol{t}_{\boldsymbol{i}}^{T}\right)^{T}$

The nodal displacement vector and the nodal force vector of member $i$ with respect to the global coordinates are denoted by $\boldsymbol{u}_{i} \in \mathbb{R}^{6}$ and $\boldsymbol{f}_{i} \in \mathbb{R}^{6}$, respectively, which have six components in the directions of six axes in Fig. 1. Then, $\boldsymbol{u}_{i}$ and $\boldsymbol{f}_{i}$ are related by the $6 \times 6$ member stiffness matrix $\boldsymbol{k}_{i}$ with respect to the global coordinates as

$\boldsymbol{f}_{i}=\boldsymbol{k}_{i} \boldsymbol{u}_{i}$

where

$\boldsymbol{k}_{i}=\frac{A_{i} E}{L_{i}} \boldsymbol{d}_{i} \boldsymbol{d}_{i}^{T}$

with $E$ being Young's modulus.

The stiffness matrices $\boldsymbol{k}_{i}$ of all members are assembled to construct the $3 n \times 3 n$ global stiffness matrix $\boldsymbol{K}$, where $3 n$ is the total number of degrees of freedom (DOFs) of nodes including the constrained DOFs at 
supports. Let $\boldsymbol{P} \in \mathbb{R}^{3 n}$ denote the nodal load vector including support reaction force. The displacement vector $\boldsymbol{U} \in \mathbb{R}^{3 n}$ is obtained by solving the stiffness (equilibrium) equation with appropriate support conditions.

The unit directional vectors $\boldsymbol{t}_{i}$ of all $m$ members are assembled to construct the $3 n \times m$ equilibrium matrix $\boldsymbol{T}$. Relation between the axial force vector $\boldsymbol{N} \in \mathbb{R}^{m}$ and $\boldsymbol{P}$ is written as

$T N=P$

Let $L_{i}$ denote the length of the $i$ th member. The axial strain vector $\varepsilon \in \mathbb{R}^{m}$ can be computed from $\boldsymbol{U}$ as

$\boldsymbol{\varepsilon}=(\operatorname{diag} \boldsymbol{L})^{-1} \boldsymbol{T}^{T} \boldsymbol{U}$

Then, the axial force $N_{i}$ of member $i$ can be expressed as

$N_{i}=\varepsilon_{i} E A_{i}$

\section{Force density method}

Let $q_{i}$ denote the force density of member $i$ defined as

$q_{i}=\frac{N_{i}}{L_{i}}$

which is the ratio of axial force to length.

If member $i$ is connected to nodes $j$ and $k$, then the components of connectivity matrix, or incidence matrix, $\boldsymbol{C} \in \mathbb{R}^{m \times n}$ is defined as follows:

$C_{i j}=-1, \quad C_{i k}=1 \quad(i=1, \ldots, m)$

By using $\boldsymbol{C}$ and the force density vector $\boldsymbol{q} \in \mathbb{R}^{m}$, the force density matrix $\boldsymbol{Q} \in \mathbb{R}^{m \times m}$ can be defined as (Zhang and Ohsaki 2015)

$\boldsymbol{Q}=\boldsymbol{C}^{T} \operatorname{diag}(\boldsymbol{q}) \boldsymbol{C}$

The diagonal element $Q_{j j}$ of $\boldsymbol{Q}$ is equal to the total amount of force densities of members connected to node $j$, and off-diagonal element $Q_{j k}(j \neq k)$ is equal to $-q_{i}$, if node $k$ is connected to node $j$ by member $i$.

Let $\boldsymbol{x} \in \mathbb{R}^{n}, \boldsymbol{y} \in \mathbb{R}^{n}$, and $\boldsymbol{z} \in \mathbb{R}^{n}$ denote the vectors of $x$-, $y$-, and $z$-coordinates, respectively, of all nodes. The axial force vector $\boldsymbol{n}_{i} \in \mathbb{R}^{3}$ of the $i$ th member connecting nodes $j$ and $k$ is defined as

$$
\begin{aligned}
\boldsymbol{n}_{i} & =N_{i} \boldsymbol{t}_{i} \\
& =N_{i}\left(\begin{array}{l}
\left(x_{k}-x_{j}\right) / L_{i} \\
\left(y_{k}-y_{j}\right) / L_{i} \\
\left(z_{k}-z_{j}\right) / L_{i}
\end{array}\right) \\
& =q_{i}\left(\begin{array}{l}
x_{k}-x_{j} \\
y_{k}-y_{j} \\
z_{k}-z_{j}
\end{array}\right)
\end{aligned}
$$

Therefore, the equilibrium equations in $x-, y$-, and $z$ directions have the following same form:

$\boldsymbol{Q x}=\boldsymbol{p}^{x}, \quad \boldsymbol{Q} \boldsymbol{y}=\boldsymbol{p}^{y}, \quad \boldsymbol{Q} \boldsymbol{z}=\boldsymbol{p}^{z}$

where $\boldsymbol{p}^{x} \in \mathbb{R}^{n}, \boldsymbol{p}^{y} \in \mathbb{R}^{n}$, and $\boldsymbol{p}^{z} \in \mathbb{R}^{n}$ are the nodal load vectors including the reactions in $x$-, $y$-, and $z$ directions, respectively. See Sheck (1974), Tibert and Pellegrino (2011), and Zhang and Ohsaki (2006) for details.

The nodes are re-ordered such that the components of free nodes precede those of fixed nodes as

$\boldsymbol{x}=\left(\begin{array}{c}\boldsymbol{x}_{\text {free }} \\ \boldsymbol{x}_{\text {fix }}\end{array}\right), \quad \boldsymbol{y}=\left(\begin{array}{c}\boldsymbol{y}_{\text {free }} \\ \boldsymbol{y}_{\text {fix }}\end{array}\right), \quad \boldsymbol{z}=\left(\begin{array}{c}\boldsymbol{z}_{\text {free }} \\ \boldsymbol{z}_{\text {fix }}\end{array}\right)$

The force density matrices in three directions are combined to $\tilde{\boldsymbol{Q}} \in \mathbb{R}^{3 n \times 3 n}$ as

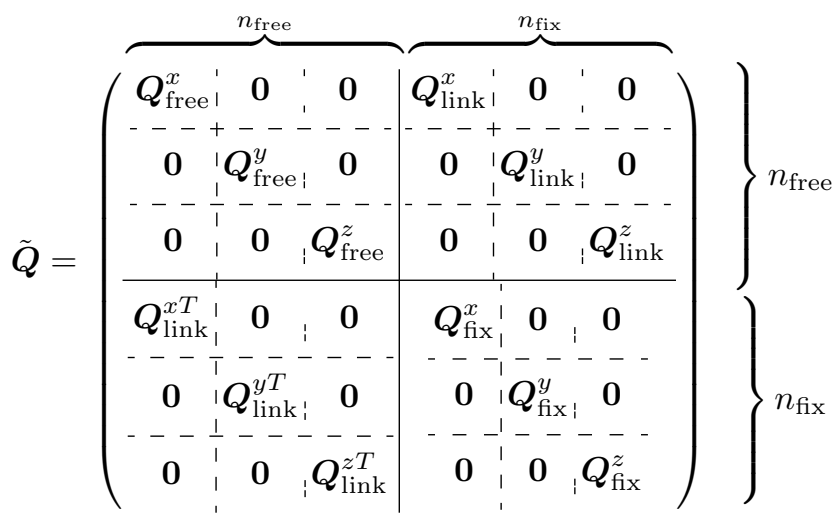

where $n_{\text {free }}$ and $n_{\text {fix }}$ are the numbers of free and fixed DOFs satisfying

$n_{\text {free }}+n_{\text {fix }}=3 n$

Remark 1 In the conventional formulation of force density method, the numbers of free and fixed nodes depend on the direction of displacement, if there exist roller supports. However, in the following formulation, the fixed node means that the location of the node is fixed in the optimization problem. Therefore, we conceive all pin supports, roller supports, and loaded nodes as fixed nodes. Therefore, the numbers of free components and the fixed components, respectively, in three directions are the same, and they are added to compute $n_{\text {free }}$ and $n_{\text {fix }}$.

The total amount of force densities for members connected to node $j$ is stored in the diagonal element $(j, j)$ of $\boldsymbol{Q}_{\text {free }}^{x}$ or $\boldsymbol{Q}_{\mathrm{fix}}^{x}$, if node $j$ is free or fixed, respectively. The value of $q_{k}$ is stored in $\boldsymbol{Q}_{\text {link }}^{x}$, if member $k$ connects a free node and a fixed node. The matrices $\boldsymbol{Q}_{\mathrm{free}}^{y}, \boldsymbol{Q}_{\mathrm{free}}^{z}, \boldsymbol{Q}_{\mathrm{fix}}^{y}, \boldsymbol{Q}_{\mathrm{fix}}^{z}, \boldsymbol{Q}_{\mathrm{link}}^{y}$, and $\boldsymbol{Q}_{\text {link }}^{z}$ are defined similarly. 
The matrices $\left(\boldsymbol{Q}_{\text {free }}^{x}, \boldsymbol{Q}_{\text {free }}^{y}, \boldsymbol{Q}_{\text {free }}^{z}\right),\left(\boldsymbol{Q}_{\mathrm{fix}}^{x}, \boldsymbol{Q}_{\text {fix }}^{y}, \boldsymbol{Q}_{\text {fix }}^{z}\right)$, and $\left(\boldsymbol{Q}_{\text {link }}^{x}, \boldsymbol{Q}_{\text {link }}^{y}, \boldsymbol{Q}_{\text {link }}^{z}\right)$ are combined to $\tilde{\boldsymbol{Q}}_{\text {free }} \in \mathbb{R}^{n_{\text {free }} \times n_{\text {free }}}$, $\tilde{\boldsymbol{Q}}_{\text {link }} \in \mathbb{R}^{n_{\text {free }} \times n_{\text {fix }}}$, and $\tilde{\boldsymbol{Q}}_{\text {fix }} \in \mathbb{R}^{n_{\text {fix }} \times n_{\text {fix }}}$, respectively, and $\tilde{\boldsymbol{Q}}$ in (13) is rewritten simply as

$\tilde{\boldsymbol{Q}}=\left(\begin{array}{cc}\tilde{\boldsymbol{Q}}_{\mathrm{free}} & \tilde{\boldsymbol{Q}}_{\text {link }} \\ \tilde{\boldsymbol{Q}}_{\text {link }}^{T} & \tilde{\boldsymbol{Q}}_{\mathrm{fix}}\end{array}\right)$

The set of equilibrium equations in (11) is combined as

$$
\left(\begin{array}{cc}
\tilde{\boldsymbol{Q}}_{\text {free }} & \tilde{\boldsymbol{Q}}_{\text {link }} \\
\tilde{\boldsymbol{Q}}_{\text {link }}^{T} & \tilde{\boldsymbol{Q}}_{\text {fix }}
\end{array}\right)\left(\begin{array}{c}
\boldsymbol{X}_{\text {free }} \\
\boldsymbol{X}_{\text {fix }}
\end{array}\right)=\left(\begin{array}{c}
\boldsymbol{P}_{\text {free }} \\
\boldsymbol{P}_{\text {fix }}
\end{array}\right)
$$

where

$$
\begin{aligned}
\boldsymbol{X}_{\text {free }} & =\left(\boldsymbol{x}_{\text {free }}^{T}, \boldsymbol{y}_{\text {free }}^{T}, \boldsymbol{z}_{\text {free }}^{T}\right)^{T}, \\
\boldsymbol{X}_{\text {fix }} & =\left(\boldsymbol{x}_{\text {fix }}^{T}, \boldsymbol{y}_{\text {fix }}^{T}, \boldsymbol{z}_{\text {fix }}^{T}\right)^{T}
\end{aligned}
$$

and $\boldsymbol{P}_{\text {free }} \in \mathbb{R}^{n_{\text {free }}}$ and $\boldsymbol{P}_{\text {fix }} \in \mathbb{R}^{n_{\text {fix }}}$ are the nodal load vectors corresponding to $\boldsymbol{X}_{\text {free }}$ and $\boldsymbol{X}_{\text {fix }}$, respectively. Note that the locations of loaded nodes are fixed in the following formulation of optimization problem; therefore, they are included in fixed nodes and $\boldsymbol{P}_{\text {free }}$ is a zero vector for any arbitrary model.

If the force densities of all members and locations of fixed nodes are assigned, then the locations of free nodes are obtained from the following set of linear equations, which is derived from (16):

$\tilde{\boldsymbol{Q}}_{\text {free }} \boldsymbol{X}_{\text {free }}=\boldsymbol{P}_{\text {free }}-\tilde{\boldsymbol{Q}}_{\text {link }} \boldsymbol{X}_{\text {fix }}$

Therefore, $\boldsymbol{X}_{\text {free }}$ is considered as function of $\boldsymbol{q}$.

Remark 2 It has been proved in Kanno (2003) that the connectivity matrix $\boldsymbol{C}$ corresponding to free nodes is non-singular, if at least one node is fixed. Therefore, if $q_{i}$ for sufficient number of members have non-zero values and coordinate of at least one node is fixed, $\boldsymbol{Q}_{\mathrm{free}}^{x}, \boldsymbol{Q}_{\mathrm{free}}^{y}$, and $\boldsymbol{Q}_{\text {free }}^{z}$ are non-singular; and accordingly, (18) has a solution.

Let $\boldsymbol{R}=\boldsymbol{P}_{\text {fix }} \in \mathbb{R}^{n_{\mathrm{fix}}}$ denote the vector of reaction forces corresponding to $\boldsymbol{X}_{\text {fix }}$. Loaded nodes are treated as fixed, and constraints are given so that the reaction force is equal to the specified external load. $\boldsymbol{R}$ is obtained from

$\boldsymbol{R}=\tilde{\boldsymbol{Q}}_{\text {link }}^{T} \boldsymbol{X}_{\text {free }}+\tilde{\boldsymbol{Q}}_{\text {fix }} \boldsymbol{X}_{\text {fix }}$

For member $i$ that connects nodes $j$ and $k$, the square of length $L_{i}$ is given as

$L_{i}^{2}=\left(\boldsymbol{X}_{k}-\boldsymbol{X}_{j}\right) \cdot\left(\boldsymbol{X}_{k}-\boldsymbol{X}_{j}\right)$

where $\boldsymbol{X}_{j} \in \mathbb{R}^{3}$ and $\boldsymbol{X}_{k} \in \mathbb{R}^{3}$ are the vectors composed of $x-, y$-, and $z$-coordinates of nodes $j$ and $k$, respectively. Note that $L_{i}^{2}$ is also a function of $\boldsymbol{q}$.

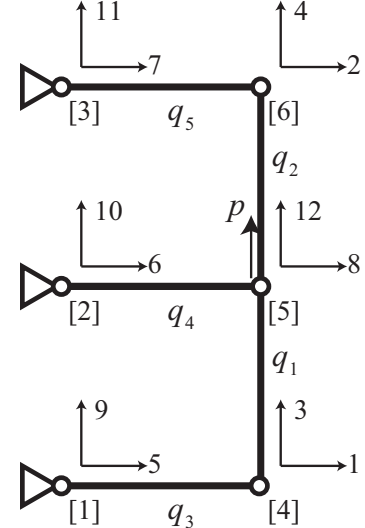

Fig. 2 Coordinate numbering of $1 \times 2$ plane grid truss.

Remark 3 As pointed out by Achtziger (2007) and Descamps and Coelho (2014), the sensitivity coefficients of length $L_{i}$ with respect to nodal coordinates are discontinuous at $L_{i}=0$. We alleviate this difficulty by avoiding the use of $L_{i}$ in the objective and constraint functions of the optimization problem; i.e., only $L_{i}^{2}$ is used.

For a simple $1 \times 2$ plane grid truss in Fig. 2, suppose a load $p$ is applied in $y$-direction at node 5 . Then, the truss has four fixed nodes 1, 2, 3, 5, and two free nodes 4, 6. Accordingly, the components of vectors $\boldsymbol{X}_{\text {free }}, \boldsymbol{X}_{\text {fix }}$, $\boldsymbol{P}_{\text {free }}, \boldsymbol{P}_{\text {free }}$, and matrices $\tilde{\boldsymbol{Q}}_{\text {free }}, \tilde{\boldsymbol{Q}}_{\text {fix }}, \tilde{\boldsymbol{Q}}_{\text {link }}$ are defined as follows:

$$
\begin{aligned}
& \boldsymbol{X}_{\text {free }}=\left(x_{4}, x_{6}, y_{4}, y_{6}\right)^{T}, \\
& \boldsymbol{X}_{\text {fix }}=\left(x_{1}, x_{2}, x_{3}, x_{5}, y_{1}, y_{2}, y_{3}, y_{5}\right)^{T} \text {, } \\
& \boldsymbol{P}_{\text {free }}=(0,0,0,0)^{T}, \\
& \boldsymbol{P}_{\text {fix }}=(0,0,0,0,0,0,0, p)^{T} \\
& \tilde{\boldsymbol{Q}}_{\text {free }}=\left(\begin{array}{cc:c}
q_{1}+q_{3} & 0 & \mathbf{0} \\
0 & q_{2}+q_{5} & \\
\hdashline \mathbf{0} & q_{1}+q_{3} & 0 \\
\hdashline & 0 & q_{2}+q_{5}
\end{array}\right)
\end{aligned}
$$

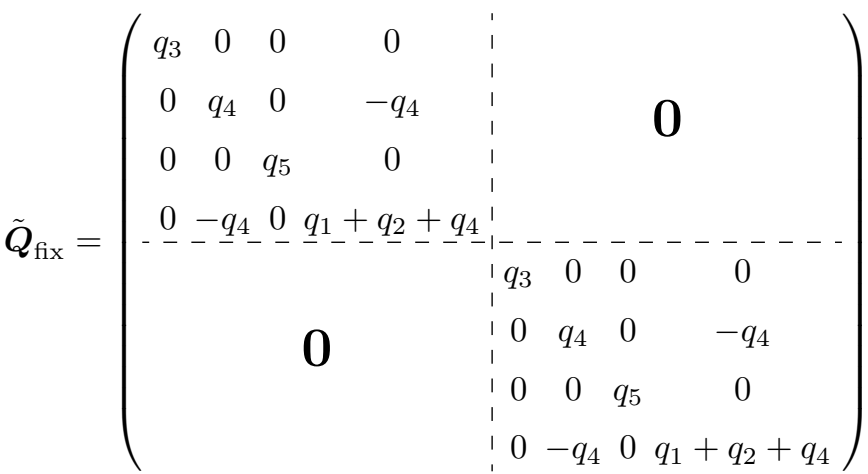




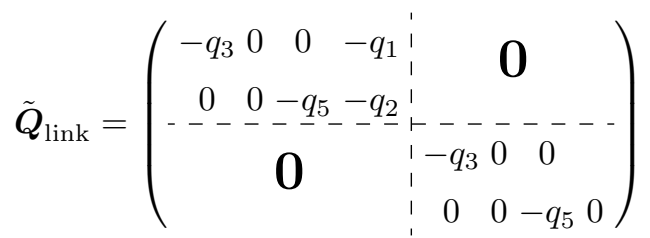

\section{Optimization problem}

Consider a problem for minimizing compliance under constraint on total structural volume. We first formulate the problem with the coordinates of free nodes and the cross-sectional areas of members as design variables, Then, the problem is regarded as a two-stage optimization problem, which is reformulated to a single-stage problem with force densities as design variables incorporating the properties of optimal solution of the lowerlevel problem.

The problem of simultaneous optimization of geometry and topology is first simply formulated as

$$
\begin{array}{ll}
\operatorname{minimize} & F(\boldsymbol{X}, \boldsymbol{A})=\sum_{i=1}^{m} \boldsymbol{u}_{i}^{T} \boldsymbol{k}_{i} \boldsymbol{u}_{i} \\
\text { subject to } & V(\boldsymbol{X}, \boldsymbol{A})=\sum_{i=1}^{m} A_{i} L_{i} \leq \bar{V} \\
A_{i}^{\mathrm{L}} \leq A_{i} \leq A_{i}^{\mathrm{U}}(i=1, \ldots, m) \\
X_{i}^{\mathrm{L}} \leq X_{i} \leq X_{i}^{\mathrm{U}}\left(i=1, \ldots, n_{\text {free }}\right)
\end{array}
$$

where ()$^{\mathrm{L}}$ and ()$^{\mathrm{U}}$ indicate lower and upper bounds, respectively, of a variable. Note that $\boldsymbol{k}_{i}$ defined in (3) is an explicit function of $\boldsymbol{A}$ and $\boldsymbol{X}$, while $\boldsymbol{u}_{i}$ is an implicit function of $\boldsymbol{A}$ and $\boldsymbol{X}$ that are obtained by solving the stiffness equation.

For fixed nodal locations, the optimal topology can be found by minimizing the compliance considering crosssectional areas as design variables; hence, the optimal cross-sectional areas can be regarded as function of $\boldsymbol{X}$. Therefore, Problem (25) can be formulated as a twostage parametric programing problem Ohsaki 1993, which is also called an implicit programming problem.

The lower-level problem is formulated as

$$
\text { minimize } F_{X}(\boldsymbol{A})
$$

$$
\begin{aligned}
& \text { subject to } V_{X}(\boldsymbol{A})=\bar{V} \\
& \qquad A_{i}^{\mathrm{L}} \leq A_{i} \leq A_{i}^{\mathrm{U}} \quad(i=1, \ldots, m)
\end{aligned}
$$

where the subscript ()$_{X}$ indicates that $\boldsymbol{X}$ is fixed. Since the optimal solution of Problem (26) is obtained for specified value of $\boldsymbol{X}$, it can be regarded as functions of $\boldsymbol{X}$, which is indicated by a hat as $\hat{\boldsymbol{A}}(\boldsymbol{X})$; accordingly, $F$ and $V$ are also functions of $\boldsymbol{X}$ only as
$\hat{F}(\boldsymbol{X})=F(\hat{\boldsymbol{A}}(\boldsymbol{X}), \boldsymbol{X})$ and $\hat{V}(\boldsymbol{X})=V(\hat{\boldsymbol{A}}(\boldsymbol{X}), \boldsymbol{X})$, respectively.

Hence, the upper-level problem is formulated as

$$
\begin{array}{ll}
\operatorname{minimize} & \hat{F}(\boldsymbol{X}) \\
\text { subject to } & \hat{V}(\boldsymbol{X})=\bar{V} \\
& X_{i}^{\mathrm{L}} \leq X_{i} \leq X_{i}^{\mathrm{U}} \quad\left(i=1, \ldots, n_{\text {free }}\right)
\end{array}
$$

It is known that the optimal solution to the lower-level problem (26) is a statically determinate truss, and all existing members have the same absolute value of stress (Hemp 1973, Achtziger et al. 1992).

Remark 4 The optimal truss is sometimes unstable with collinear two members connected to a node. However, even in such case, the truss is statically determinate in the sense that the axial forces of members are uniquely determined irrespective of the size of members. Unstable optimal solutions are often found when only a single loading condition is considered. Unstable solutions can be stabilized by fixing pin joints or adding a thin member after optimization, when we do not consider member buckling. Assigning fictitious nominal forces is also effective as proposed by Descamps and Coelho (2014). Since compatibility conditions between strains and displacements are neglected in the problem formulation, statical indeterminacy should be checked for the solution, and the solution should be rejected if it happens to be statically indeterminate.

Let $\bar{\sigma}$ denote the absolute value of stress for existing members of the optimal solution of Problem (26). In the following, ( ) indicates a function of $\boldsymbol{q}$ only. Using $\bar{\sigma}, A_{i}$ is expressed in terms of $q_{i}$ as

$$
\begin{aligned}
\tilde{A}_{i}(\boldsymbol{q}) & =\frac{\left|N_{i}\right|}{\bar{\sigma}} \\
& =\frac{\left|q_{i}\right| L_{i}}{\bar{\sigma}}
\end{aligned}
$$

Since $\boldsymbol{X}$ is computed from $\boldsymbol{q}$ using (18), all variables are defined by $\boldsymbol{q}$. The volume $V_{i}$ of member $i$ is defined using $\boldsymbol{q}$ as

$\tilde{V}_{i}(\boldsymbol{q})=\frac{\left|q_{i}\right| L_{i}^{2}}{\bar{\sigma}}$

Let $S_{i}$ denote the strain energy of member $i$. Using (28), $S_{i}$ is written as

$$
\begin{aligned}
\tilde{S}_{i}(\boldsymbol{q}) & =\frac{A_{i} L_{i}}{2 E} \bar{\sigma}^{2} \\
& =\frac{\bar{\sigma}\left|q_{i}\right| L_{i}^{2}}{2 E}
\end{aligned}
$$


Therefore, the compliance $F$ is obtained as

$$
\begin{aligned}
\tilde{F}(\boldsymbol{q}) & =2 \sum_{i=1}^{m} \frac{\bar{\sigma}\left|q_{i}\right| L_{i}^{2}}{2 E} \\
& =\sum_{i=1}^{m} \frac{\bar{\sigma}\left|q_{i}\right| L_{i}^{2}}{E}
\end{aligned}
$$

It is seen from (28) that $\bar{\sigma}$ can be regarded as a scaling parameter for $A_{i}$ of a statically determinate truss, for which $N_{i}$ is independent of $A_{i}$. From (29) and (31), the product of $V$ and $F$ is computed as

$$
\begin{aligned}
\tilde{V} \tilde{F} & =\sum_{i=1}^{m} \frac{q_{i}^{2} L_{i}^{4}}{E} \\
& =\sum_{i=1}^{m} \frac{N_{i}^{2} L_{i}^{2}}{E}
\end{aligned}
$$

which means that $\tilde{V} \tilde{F}$ is independent of $\bar{\sigma}$. Hence, the total volume of members can be calculated after the optimal solution is obtained by minimizing the compliance with arbitrary positive value of $\bar{\sigma}$.

Since $\boldsymbol{A}$ and $\boldsymbol{X}$ are functions of $\boldsymbol{q}$, and the volume constraints can be ignored, the simultaneous optimization problem (25) is reformulated as

$$
\begin{array}{ll}
\text { minimize } & \tilde{F}(\boldsymbol{q})=\sum_{i=1}^{m} \frac{\bar{\sigma}\left|q_{i}\right| L_{i}^{2}}{E} \\
\text { subject to } & R_{i}(\boldsymbol{q})=\bar{R}_{i} \quad(i \in \mathcal{R}) \\
& q_{i}^{\mathrm{L}} \leq q_{i} \leq q_{i}^{\mathrm{U}} \quad(i=1, \ldots, m)
\end{array}
$$

where $q_{i}^{\mathrm{L}}$ and $q_{i}^{\mathrm{U}}$ are the lower and upper bounds of $q_{i}$, respectively, and $\mathcal{R}$ is the set of indices of reaction components that are to be specified.

This way, the number of variables is reduced to $m$. In contrast, in the formulation by Descamps and Coelho (2014), the number of variables is $3 m+d$, where $d$ is the number of variables of nodal coordinates. It is pointed out by Rojas-Labanda and Stolpe (2015) that the formulation using simultaneous analysis and design (SAND) for topology optimization demands more computational cost than the nested formulation for largescale problems. Therefore, it is important to formulate a problem with small number of variables.

Although Problem (33) is a simple NLP problem with $\boldsymbol{q}$ as the design variable, $\left|q_{i}\right|$ is non differentiable at $q_{i}=0$. A standard approach to prevent this discontinuity is to use auxiliary variables $q_{i}^{+}$and $q_{i}^{-}$as

$q_{i}=q_{i}^{+}-q_{i}^{-}, \quad\left|q_{i}\right|=q_{i}^{+}+q_{i}^{-}, \quad q_{i}^{+} \geq 0, \quad q_{i}^{-} \geq 0$
However, we use the smoothing approximation, because our purpose is to obtain the optimal topology, geometry, and cross-sectional areas by considering only $\boldsymbol{q}$ as design variables. Accordingly, $\left|q_{i}\right|$ is approximated as

$\left|q_{i}\right|=\sqrt{q_{i}^{2}+c}$

where $c$ is a sufficiently small positive number. The compliance is approximated as

$\tilde{F}^{a}(\boldsymbol{q})=\sum_{i=1}^{m} \frac{\bar{\sigma} L_{i}^{2} \sqrt{q_{i}^{2}+c}}{E}$

\section{Sensitivity analysis}

We use sequential quadratic programming, which is a gradient-based approach of NLP. Therefore, sensitivity coefficients of objective and constraint functions are needed for reduction of computation time.

Differentiation of (36) with respect to $q_{l}$ leads to

$\frac{\partial \tilde{F}^{a}(\boldsymbol{q})}{\partial q_{l}}=\frac{\bar{\sigma} q_{l} L_{l}^{2}}{E \sqrt{q_{l}^{2}+c}}+\sum_{i=1}^{m}\left(\frac{\bar{\sigma} \sqrt{q_{i}^{2}+c}}{E} \cdot \frac{\partial L_{i}^{2}}{\partial q_{l}}\right)$

From (20), the sensitivity coefficient of $L_{i}^{2}$ with respect to $q_{l}$ is obtained as

$\frac{\partial L_{i}^{2}}{\partial q_{l}}=2\left(\boldsymbol{X}_{j}-\boldsymbol{X}_{k}\right) \cdot \frac{\partial\left(\boldsymbol{X}_{j}-\boldsymbol{X}_{k}\right)}{\partial q_{l}}$

Differentiation of (18) and (19) with respect to $q_{l}$ leads to

$\tilde{\boldsymbol{Q}}_{\text {free }} \frac{\partial \boldsymbol{X}_{\text {free }}}{\partial q_{l}}=-\frac{\partial \tilde{\boldsymbol{Q}}_{\text {free }}}{\partial q_{l}} \boldsymbol{X}_{\text {free }}-\frac{\partial \tilde{\boldsymbol{Q}}_{\text {link }}}{\partial q_{l}} \boldsymbol{X}_{\text {fix }}$

$\frac{\partial \boldsymbol{R}}{\partial q_{l}}=\frac{\partial \tilde{\boldsymbol{Q}}_{\text {link }}^{T}}{\partial q_{l}} \boldsymbol{X}_{\text {free }}+\tilde{\boldsymbol{Q}}_{\text {link }}^{T} \frac{\partial \boldsymbol{X}_{\text {free }}}{\partial q_{l}}+\frac{\partial \tilde{\boldsymbol{Q}}_{\text {fix }}}{\partial q_{l}} \boldsymbol{X}_{\text {fix }}$

For the current value of $\boldsymbol{q}$ during optimization, the set of linear equations (39) is solved for $\partial \boldsymbol{X}_{\text {free }} / \partial q_{l}$, which is incorporated successively into (37), (38), and (40) to obtain the sensitivity coefficients of the objective and constraint functions.

\section{Improvement of optimal solution}

Since the optimal solution to Problem (33) may include many overlapped nodes and members, and the nodal 
positions are obscure, we further optimize the crosssectional areas of members and the nodal coordinates. The following problem is solved with fixed topology:

$\operatorname{minimize} F(\boldsymbol{X}, \boldsymbol{A})=\sum_{i=1}^{m^{*}} \frac{N_{i}^{2} L_{i}}{E A_{i}}$

$$
\begin{aligned}
\text { subject to } & \sum_{i=1}^{m^{*}} A_{i} L_{i} \leq \bar{V} \\
& X_{i}^{\mathrm{L}} \leq X_{i} \leq X_{i}^{\mathrm{U}} \quad\left(i=1, \ldots, n_{\text {free }}^{*}\right) \\
& A_{i}^{\mathrm{L}} \leq A_{i} \leq A_{i}^{\mathrm{U}} \quad\left(i=1, \ldots, m^{*}\right)
\end{aligned}
$$

where $m^{*}$ and $n_{\text {free }}^{*}$ are the numbers after unifying overlapped nodes and members in the optimal solution to Problem (33). The same symbols as the previous section are used for variables for convenience.

The lower bound $A_{i}^{\mathrm{L}}$ for $A_{i}$ is a sufficiently small positive value, and the member with $A_{i}=A_{i}^{\mathrm{L}}$ is eliminated after optimization. Since we do not have to expect existence of melting nodes in this re-optimization process, upper and lower bounds $X_{i}^{\mathrm{U}}$ and $X_{i}^{\mathrm{L}}$, respectively, may be close to the initial value of $X_{i}$ that is assigned based on the optimal solution of Problem (33). Finite difference method is used for computing sensitivity coefficients, because Problem (41) is solved only once.

\section{Numerical examples}

We present three numerical examples to confirm the efficiency of the optimization method proposed in the previous sections. A sequential quadratic programming (SQP) implemented in the library SNOPT Ver 7.2 (Gill et al. 2002) is used for solving NLP problems. Units are omitted in the following examples, because they are not important in this research.

Young's modulus is 1.0 for comparison to the results in Achtziger (2007) and Descamps and Coelho (2014). The values of $c$ in (35) and $\bar{\sigma}$ are $1.0 \times 10^{-6}$ and 1.0, respectively, for all examples. Let $\overline{\boldsymbol{q}}=\left(\bar{q}_{1}, \ldots, \bar{q}_{i}\right)^{T}$ denote the vector of force density of the initial regular truss with uniform cross-sectional areas. The lower and upper bounds for $q_{i}$ are given as $q_{i}^{\mathrm{L}}=q_{i}-\Delta q$ and $q_{i}^{\mathrm{U}}=q_{i}+\Delta q$, respectively. The initial values of force density for optimization are randomly provided within the range $\left[\bar{q}_{i}-\delta q, \bar{q}_{i}+\delta q\right]$. We choose the best solution out of 100 solutions obtained using 100 different random seeds.

\subsection{Example $1: 3 \times 2$ truss with 27 members}

The first example is optimization of a plane truss model. The initial ground structure is a $3 \times 2$ rectangular grid as

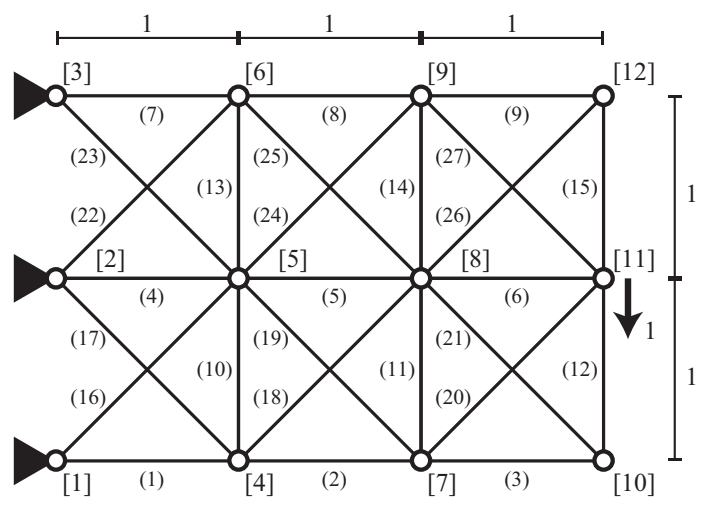

Fig. 3 Initial ground structure of a $3 \times 2$ plane grid.

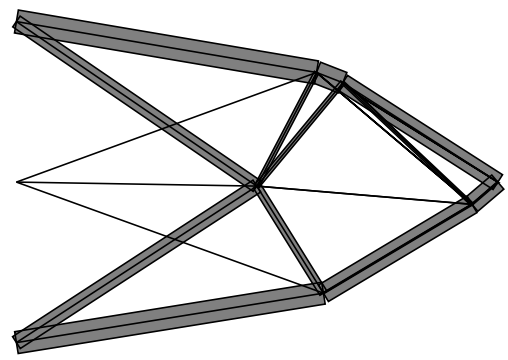

Fig. 4 Optimal solution of $3 \times 2$ grid obtained by solving Problem (33) $\left(F^{*}=8.316\right)$.

Table 1 Nodal location of optimal solution of $3 \times 2$ grid.

\begin{tabular}{ccc}
\hline node & $x$ & $y$ \\
\hline 1 & 0.0 & 0.0 \\
2 & 0.0 & 1.0 \\
3 & 0.0 & 2.0 \\
4 & 1.912 & 0.308 \\
5 & 1.498 & 0.975 \\
6 & 1.870 & 1.683 \\
7 & 2.839 & 0.859 \\
8 & 2.838 & 0.861 \\
9 & 2.033 & 1.620 \\
10 & 2.841 & 0.864 \\
11 & 3.0 & 1.0 \\
12 & 2.830 & 0.868 \\
\hline & &
\end{tabular}

shown in Fig. 3 with 27 members and 12 nodes including supports. Note that the intersecting diagonal members are not connected with each other. The truss is pinsupported at nodes 1, 2, 3, and a downward unit load is applied at node 11 . Nodal coordinates are optimized fixing the locations of these four nodes with parameter values $\Delta q=1000.0$ and $\delta q=5.0$.

The best optimal solution to Problem (33) is obtained as shown in Fig. 4 , where $\tilde{F}^{a}$ and $\tilde{V}^{a}$ have the same value 9.131 . The values of $\tilde{F}$ and $\tilde{V}$ without smoothing are 9.119. The compliance $F^{*}$ corresponding to the total volume 10.0 is computed as $F^{*}=\tilde{F} \tilde{V} / 10.0=$ 8.316 , which is almost equal to $F^{*}=8.307$ obtained by Achtziger (2007). The nodal locations of optimal solution are listed in Table 1 . Note that nodes 7, 8, 10, and 
Table 2 Force density, cross-sectional area, and length of members of optimal solution of $3 \times 2$ grid.

\begin{tabular}{cccccc}
\hline member & \multicolumn{2}{c}{ nodes } & $q_{i}$ & $A_{i}$ & $L_{i}$ \\
\hline 1 & 1 & 4 & -0.509 & 0.986 & 1.937 \\
2 & 4 & 7 & -0.050 & 0.054 & 1.079 \\
3 & 7 & 10 & 1.736 & 0.003 & 0.002 \\
4 & 2 & 5 & 0.000 & 0.001 & 1.499 \\
5 & 5 & 8 & 0.000 & 0.001 & 1.345 \\
6 & 8 & 11 & -3.874 & 0.823 & 0.213 \\
7 & 3 & 6 & 0.546 & 1.036 & 1.897 \\
8 & 6 & 9 & 5.609 & 0.981 & 0.175 \\
9 & 9 & 12 & 0.202 & 0.024 & 0.118 \\
10 & 4 & 5 & 0.465 & 0.365 & 0.785 \\
11 & 7 & 8 & 4.635 & 0.006 & 0.001 \\
12 & 10 & 11 & -0.010 & 0.002 & 0.212 \\
13 & 5 & 6 & -0.266 & 0.213 & 0.800 \\
14 & 8 & 9 & 0.119 & 0.132 & 1.106 \\
15 & 11 & 12 & 0.013 & 0.013 & 1.030 \\
16 & 1 & 5 & -0.351 & 0.628 & 1.788 \\
17 & 4 & 2 & 0.000 & 0.002 & 2.034 \\
18 & 4 & 8 & -0.793 & 0.856 & 1.079 \\
19 & 7 & 5 & 0.000 & 0.001 & 1.346 \\
20 & 7 & 11 & -0.254 & 0.054 & 0.213 \\
21 & 10 & 8 & -5.423 & 0.005 & 0.001 \\
22 & 2 & 6 & 0.000 & 0.002 & 1.991 \\
23 & 5 & 3 & 0.319 & 0.579 & 1.816 \\
24 & 5 & 9 & -0.265 & 0.222 & 0.838 \\
25 & 8 & 6 & 0.009 & 0.011 & 1.269 \\
26 & 8 & 12 & 0.011 & 0.011 & 0.988 \\
27 & 11 & 9 & 0.680 & 0.780 & 1.148 \\
\hline & & & & &
\end{tabular}

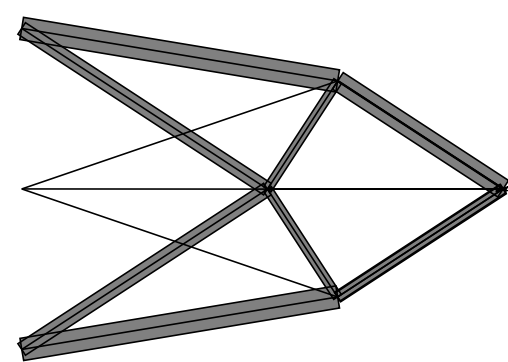

Fig. 5 Fourth best solution among 100 optimal solutions of Problem (33) of $3 \times 2$ grid $\left(F^{*}=8.347\right)$.

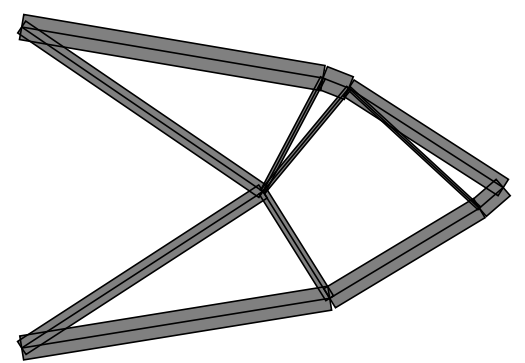

Fig. 6 Solution of re-optimization of $3 \times 2$ grid $(F=8.312)$.

12 converged to an almost the same place; i.e., three nodes melted.

The values of $q_{i}, A_{i}$, and $L_{i}$ for all members of optimal solution are listed in Table 2 . It is seen from the table that members 3,11 , and 21 that connect pairs of
Table 3 Maximum, median, minimum, average values, and standard deviation of function values for Examples 1, 2, and 3 for 100 solutions, which are scaled for comparison to existing results.

\begin{tabular}{lccc}
\hline & Ex. 1 $\left(F^{*}\right)$ & Ex. $2\left(F^{*}\right)$ & Ex. 3 $(\tilde{V})$ \\
\hline max & 10.227 & $7.850 \times 10^{6}$ & 4015.431 \\
median & 9.095 & 640.150 & 54.927 \\
min & 8.316 & 118.994 & 54.544 \\
average & 9.218 & $9.271 \times 10^{4}$ & 125.171 \\
std. dev. & 0.549 & $7.875 \times 10^{5}$ & 435.744 \\
\hline
\end{tabular}

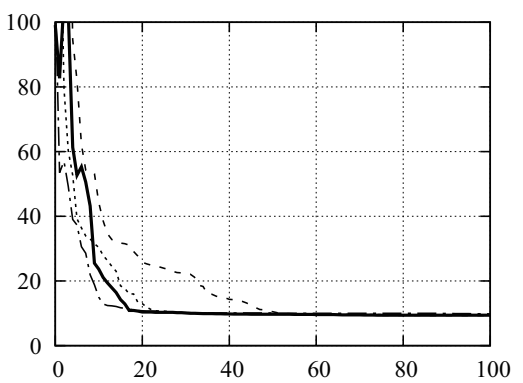

Fig. 7 Iteration history of merit function for four best solutions of $3 \times 2$ grid.

nodes $(7,10),(7,8)$, and $(8,10)$, respectively, have small member length. Since $\Delta q$ has a sufficiently large value 1000, no bound constraint is active for $q_{i}$. Note again that, as seen from (22) and (24) for the simple example truss in Fig. 2, a diagonal term of the force density matrix is the sum of the force densities of the members connected to the node. Therefore, even some force densities are zero, it does not mean that the matrix is singular. In Table 2, the force densities of members 4, 5, and 19 have very small absolute values; however, they are not exactly equal to zero. Furthermore, members $10,13,16,23$, and 24 connected to node 5 have nonzero force densities.

Iteration history of the merit function is plotted in Fig. 7 for the four best solutions of the $3 \times 2$ grid. Merit function is the objective function with penalty terms of violated constraints, and it is used for QP subproblem and line search in SQP. Therefore, almost monotonic reduction of this function indicates a good convergence of the algorithm.

The maximum, median, minimum, average values, and standard deviation of $F^{*}$ for 100 different solutions are listed in the second column of Table $3 . F^{*}$ varies around 10 with small standard deviation, which confirms the convergence property of the proposed method. Note that almost all solutions, which have vanishing members and melting nodes, are obtained without divergence in the optimization process. Moreover, the computation time for each optimization is about three seconds using a PC with Intel Core i7 processor, which implies possible application to complex models. Although 
the solutions from different random seed do not converge to the same optimal solution, it is important to note that various nearly optimal solutions with different topology can be found using the proposed method. Fig. 5 shows the fourth best solution among 100 solutions. Note that the truss in Fig. 5 is symmetric, although the best solution in Fig. 3 is asymmetric.

Next, the solution in Fig. 3 is re-optimized solving Problem (41) with the upper-bound volume $\bar{V}=10.0$. The four nodes 7, 8, 10, and 12 are combined to a single node, and node 2 and the three thin members connected to node 2 are removed before re-optimization. Bound constraints are given for the nodal coordinates; however, they are not active at the optimal solution. The upper bound $A_{i}^{\mathrm{U}}$ for $A_{i}$ is sufficiently large, and the lower bound $A_{i}^{\mathrm{L}}$ is 0.001 . The optimal solution is shown in Fig. 6, which is almost the same as the solution in Fig. 4. Note that the members with $A_{i}=A_{i}^{\mathrm{L}}$ are removed in Fig. 4. The compliance $F^{*}$ is slightly reduced to 8.312. Therefore, influence of the re-optimization is trivial and the proposed method leads to a sufficiently converged solution.

\subsection{Example $2: 6 \times 1$ truss with 31 members}

Consider next a $6 \times 1$ plane grid truss as shown in Fig. 8 . The truss has 31 members and 14 nodes including supports. Note that the left and right supports are pin and roller, respectively, and other lower nodes are subjected to downward unit loads. Throughout the optimization process, the locations of these seven nodes are fixed.

Since the $y$-coordinates of all fixed and loaded nodes are 0 , there exists an obvious solution for (18), where all $y$-coordinates vanish. To prevent this difficulty, $y$ coordinates of the loaded nodes are modified from 0.0 to -0.1 . Note that this modification does not have any effect on the final optimal solution after re-optimization, if an appropriate optimal topology is found by solving Problem (33).

The best optimal solution obtained from 100 different random seed is illustrated in Fig. 9, where $\tilde{F}^{a}$ and $\tilde{V}^{a}$ have the same value 34.520 . The values of $\tilde{F}$ and $\tilde{V}$ without smoothing are 34.496 . The compliance $F^{*}$ corresponding to the total volume 10.0 is computed as $F^{*}=\tilde{F} \tilde{V} / 10.0=118.994$, which is smaller than $F^{*}=122,477$ obtained by Achtziger (2007), because the $y$-coordinates of loaded nodes are modified. It is seen from Fig. 9 that no melting node exists in this example. Although $\Delta q=100.0$, which is $1 / 10$ of Example 1 , no bound constraint is active for $q_{i}$ at the optimal solution; however, the bounds are needed for improving convergence of solutions. The properties of function values of 100 different optimal solutions are listed in the

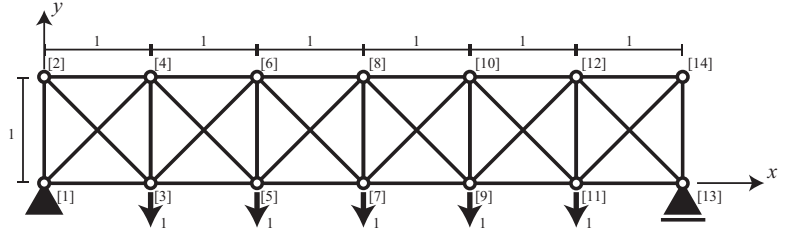

Fig. 8 Initial ground structure of a $6 \times 1$ plane grid.

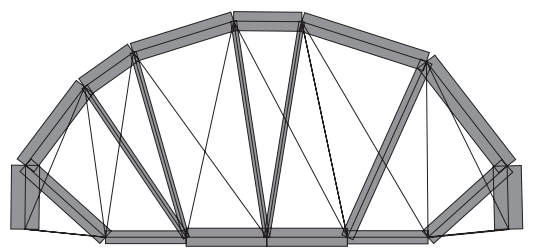

Fig. 9 Optimal solution of $6 \times 1$ grid obtained by solving Problem (33) $\left(F^{*}=118.994\right)$.

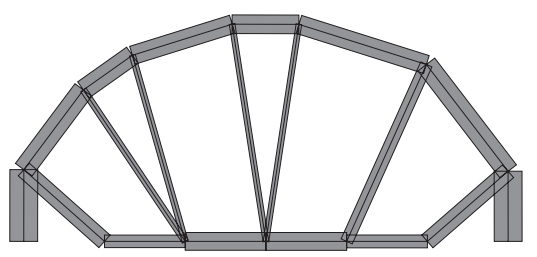

Fig. 10 Solution of re-optimization of $6 \times 1$ grid $(F=$ 122.411).

third column of Table 3. Note that solution sometimes diverges in this example; however, the median value is sufficiently small.

The truss in Fig. 9 is re-optimized to obtain the solution in Fig. 10, which is a little different from the solution in Fig. 9. Note that $y$-coordinates of the loaded nodes are 0.0 in this process. The value of compliance $F^{*}$ for $\bar{V}=10.0$ is $=122.411$, which is slightly smaller than 122.477 in Achtziger (2007). However, due to the elimination of thin members, this optimal solution is globally unstable with a zero-energy mechanism mode in $x$-direction. To prevent such instability, some members, e.g., those connecting nodes $(1,3)$ and $(11,13)$ may be left without removing.

\subsection{Example 3 : L-shaped frame}

Finally, the L-shaped three-dimensional truss in Fig. 11 is optimized. This model has been studied in Descamps and Coelho (2014) for stress constrained optimization problem. Since the compliance optimization problem under single loading condition can be reformulated as a stress constrained problem or a limit analysis problem, the optimal solution obtained using the proposed method is expected to be the same as the solution in Descamps and Coelho (2014) under appropriate parameter values. 


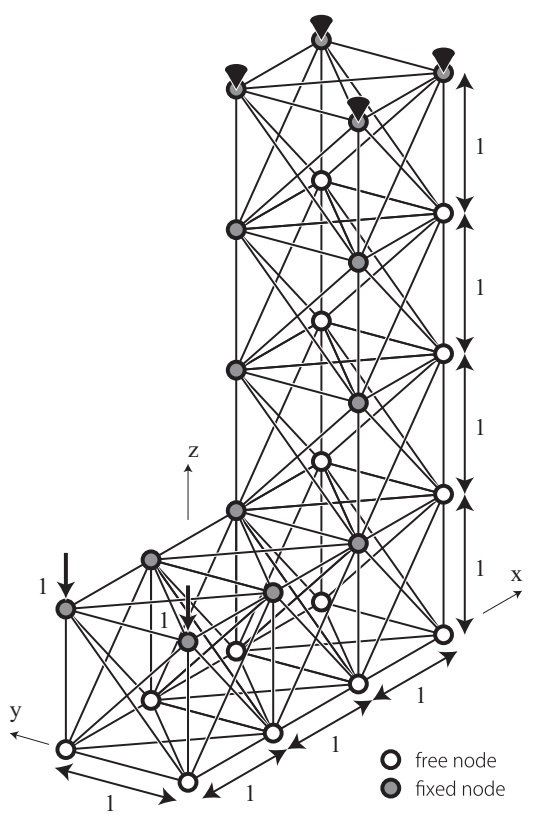

Fig. 11 Initial ground structure of an L-shaped truss.

The initial ground structure is an L-shaped truss with 28 nodes and 108 members as shown in Fig. 11. The four upper nodes are pin-supported. Each of two upper tips of the truss is subjected to unit load in negative $z$-direction.

To compare the results with those in Descamps and Coelho (2014), $x-, y$-, and $z$-coordinates of the rightmost and lower nodes, which are indicated by blank circles, are considered as design variables, and the bounding box is set as $1 \times 1 \times 1$ unit cube around the initial position in Fig. 11. The values of $\Delta q$ and $\delta q$ are 100.0 and 1.0 , respectively.

The best optimal solution obtained from 100 different random seed is shown in Fig. 12, where $\tilde{F}=54.544$. It is seen from the figure that two pairs of lower nodes turned out to be coalescent as a result of optimization. The properties of function values of 100 different optimal solutions are listed in the last column of Table 3. The value of $V$ exceeds 1000 in only two cases; therefore, no severe divergence occurs in this example. Average computation time is 16 seconds for obtaining an optimal solution.

Although 66 members in Fig. 12 are thin enough to be neglected, the number of members are decreased by only 40 to maintain stability of the truss of the ground structure for re-optimization process Note that the remaining 26 members vanish after re-optimization. The solution with $\tilde{F}=54.276$ as shown in Fig. 13 is obtained by re-optimization. Note that the objective value by Descamps and Coelho (2014) is 54.098.

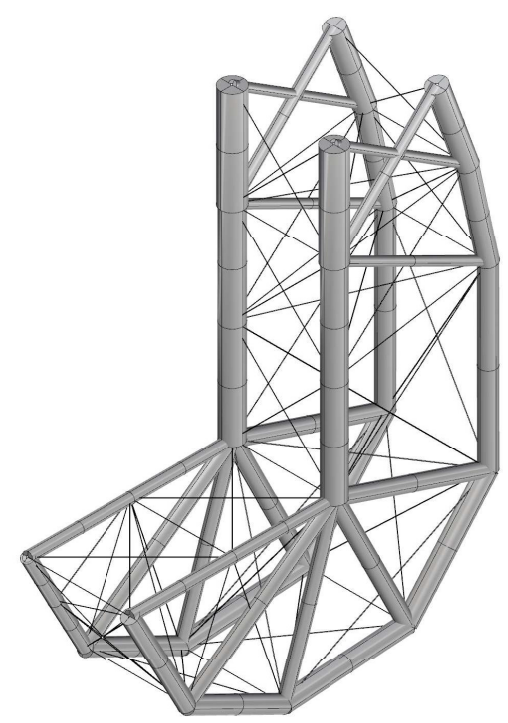

Fig. 12 Optimal solution of an L-shaped truss obtained by solving Problem (33) $(\tilde{F}=54.544)$.

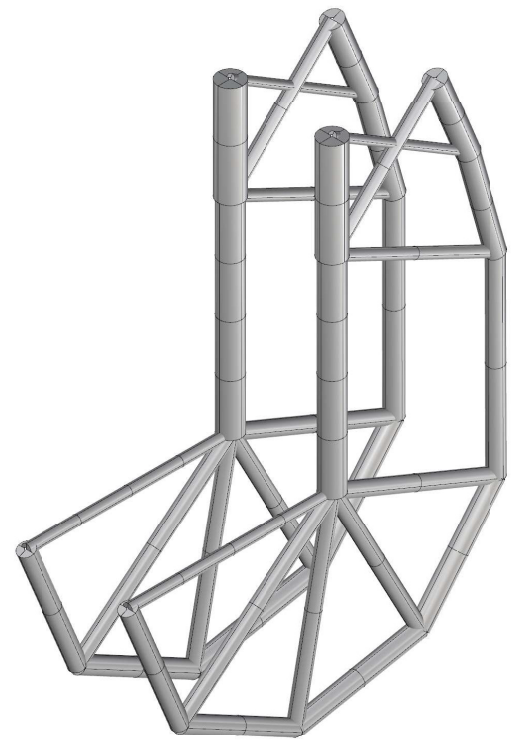

Fig. 13 Solution of re-optimization of L-shaped truss $(F=$ 54.276).

\section{Conclusions}

A new method of simultaneous optimization of geometry and topology has been presented for plane and spatial trusses. The conclusions obtained from this study are summarized as follows:

1. The difficulty in simultaneous optimization of geometry and topology can be successfully avoided using force density as design variable. The nodal coordinates are considered as functions of force densities, where the loaded nodes are also included in the fixed 
nodes, for which the coordinates are to be assigned. Regularity of the force density matrix to obtain the nodal coordinates is ensured, if the location of at least one node is fixed; however, a plane truss degenerates to a line, if all the fixed nodes have the same coordinate value in a direction; e.g., $y$-coordinates of all fixed nodes are 0 .

2. The compliance and structural volume are expressed as functions of force density only, using the fact that the optimal truss is statically determinate with the same absolute value of stress in existing members. This way, the number of design variables can be reduced to $m$, which is the number of members, even for the problem including geometry optimization. In contrast, in the formulation by Descamps and Coelho (2014), the number of variables is $3 m+d$, where $d$ is the number of variables of nodal coordinates. As pointed out by Rojas-Labanda and Stolpe (2015), it is important to formulated a problem with small number of variables.

3. Discontinuity of the sensitivity coefficient of crosssectional area with respect to the force density can be successfully avoided using smoothing function. Since the nodal locations and cross-sectional areas are re-optimized by solving a simple NLP problem, small errors such as existence of thin members do not have any effect on the final optimal solution.

4. Accuracy of the optimal solution has been verified through two examples of plane truss and an example of three-dimensional truss that have been studied in the existing literatures. Although the optimal topologies are different from those in existing studies, the differences in objective values are sufficiently small. It should be noted that various nearly optimal solutions can be found using the proposed method.

\section{Acknowledgments}

This work is partially supported by JSPS KAKENHI No. $16 \mathrm{H} 03014$.

\section{References}

W. Achtziger, On simultaneous optimization of truss geometry and topology, Struct. Multidisc. Optim., Vol. 33, pp. 285-304, 2007.

W. Achtziger, M. P. Bendsøe, A. Ben-Tal, and J. Zowe, Equivalent displacement based formulations for maximum strength truss topology design, Impact of Comput. Sci. Eng., Vol. 4(4), pp. 315-345, 1992.
M. P. Bendsøe and O. Sigmund, Topology Optimization: Theory, Methods and Applications, Springer, 2003.

B. Descamps and R. F. Coelho, The nominal force method for truss geometry optimization incorporating stability considerations, Int. J. Solids Struct. Vol. 51, pp. 2390-2399, 2014.

W. Dobbs and L. P. Felton, Optimization of truss geometry, J. Struct. Div. ASCE, Vol. 95(ST10), pp. 21052119, 1969.

W. R. Dorn, R. Gomory and H. Greenberg. Automatic design of optimal structures, J. de Mecanique, Vol. 3, pp. 25-52, 1964.

P. E. Gill, W. Murray and M. A. Saunders, SNOPT: An SQP algorithm for large-scale constrained optimization, SIAM J. Opt., Vol. 12, pp. 979-1006, 2002.

X. Guo, W. Liu and H. Li, Simultaneous shape and topology optimization of truss under local and global stability constraints, Acta Mechanica Solida Sinca, Vol. 16(2), pp. 95-101, 2003.

T. Hagishita and M. Ohsaki, Topology optimization of trusses by growing ground structure approach, Struct. Multidisc. Optim., Vol. 37(4), pp. 377-393, 2009.

W. S. Hemp, Optimum Structures, Clarendon Press, Oxford, UK, 1973.

Y. Kanno and M. Ohsaki, Minimum principle of complementary energy of cable networks by using secondorder cone programming, Int. J. Solids and Struct., Vol. 40, No. 17, pp. 4437-4460, 2003.

G.-C. Luh and C.-Y. Lin, Optimal design of truss structures using ant algorithm, Struct. Multidisc. Optim., Vol. 36, pp. 365-379, 2008.

J. J. McKeown, Growing optimal pin-jointed frames, Struct. Opt., Vol. 15, pp. 92-100, 1998.

M. Ohsaki, Simultaneous optimization of topology and geometry of a regular plane truss, Comput. \& Struct., Vol. 66(1), pp. 69-77, 1998.

M. Ohsaki, Optimization of Finite Dimensional Structures, CRC Press, 2010.

M. Ohsaki and J. S. Arora, A direct application of higher order parametric programming techniques to structural optimization, Int. J. Numer. Meth. Engng, Vol. 36, pp. 2683-2702, 1993.

S. Rojas-Labanda and M. Stolpe, Benchmarking optimization solvers for structural topology optimization, Struct. Multidisc. Optim., Vol. 52, pp. 527-547, 2015.

H.-J. Schek, The force density method for form finding and computation of general networks, Comput. Methods Appl. Mech. Eng., Vol. 3, pp. 115-134, 1974.

M. Stolpe, On the reformulation of topology optimization problems as linear or convex quadratic mixed 0-1 programs, Opt. Eng., Vol. 8, pp. 163-192, 2007. 
A. G. Tibert and S. Pellegrino, Review of form-finding methods for tensegrity structures, Int. J. Space Structures, Vol. 26(3), pp. 241-255, 2011.

Y. Yang and C. K. Soh, Automated optimum design of structures using genetic programming, Comp. Struct., Vol. 80, pp. 1537-1546, 2002.

J. Y. Zhang and M. Ohsaki, Tensegrity Structures: Form, Stability, and Symmetry, Mathematics for Industry 6, Springer, 2015.

J. Y. Zhang and M. Ohsaki, Adaptive force density method for form-finding problem of tensegrity structures, Int. J. Solids and Struct., Vol. 43, No. 18-19, pp. 5658-5673, 2006. 